\title{
Expression of angiogenic growth factors in laryngeal carcinoma
}

\author{
TIRUPATI S. KORAMPALLI and NICHOLAS D. STAFFORD \\ Postgraduate Medical Institute, University of Hull, Castle Hill Hospital, Cottingham, Hull HU16 5JQ, UK
}

Received April 9, 2013; Accepted July 19, 2013

DOI: $10.3892 / \mathrm{mco} .2013 .179$

\begin{abstract}
Over 2,200 cases of carcinoma of the larynx are diagnosed in the UK annually, with an overall 5-year survival rate of $67 \%$. Angiogenesis is vital for the growth and metastasis of solid tumours and the expression of key angiogenesis-related proteins has been shown to be of prognostic significance. In this study we reported the expression of key angiogenesis-related factors, selected from a pilot array study, in a cohort of laryngeal tumours and associated metastatic lymph nodes. Forty patients diagnosed with squamous cell carcinoma of the larynx were recruited. Tissue specimens were obtained intra-operatively, prior to chemo- and/or radiotherapy, from the tumours and secondary lymph nodes. The patient group comprised 32 men and 8 women with a mean age of 68 years (range, 51-89 years). The relative expression of the angiogenesis-related proteins angiogenin, interleukin (IL)-8, tissue inhibitor of metalloproteinases-1 (TIMP-1), vascular endothelial growth factor (VEGF), fibroblast growth factor (FGF)-basic and insulin-like growth factor binding protein 3 (IGFBP3) was determined in the tissue lysates by ELISA. The expression of angiogenin was higher in early-stage compared with late-stage tumours $(\mathrm{P}=0.034)$ and the expression of IGFBP3 was higher in tumours compared with the metastatic lymph nodes $(\mathrm{P}=0.016)$. No statistically significant differences were recorded for VEGF, FGF, TIMP-1 or IL- 8 between tumour stages or primary tumours and lymph nodes. To the best of our knowledge, this study was the first to investigate multiple angiogenic factors in the lysates of laryngeal carcinomas and metastatic nodes and identified angiogenin and IGFBP3 as factors possibly involved in tumour progression. A greater understanding of their function may offer novel prognostic and/or therapeutic options.
\end{abstract}

\section{Introduction}

Laryngeal squamous cell carcinoma (LSCC) accounts for $>95 \%$ of all laryngeal malignancies and is the most common

Correspondence to: Dr Tirupati S. Korampalli, Postgraduate Medical Institute, University of Hull, Castle Hill Hospital, Cottingham, Hull HU16 5JQ, UK

E-mail:korampalli@gmail.com

Key words: larynx, tumour, growth, angiogenesis
SCC of the head and neck (1,2). The highest incidence of this malignancy is observed in the fifth and sixth decades of life (3) and it represents $10 \%$ of malignancies in men and $4 \%$ in women worldwide. Despite recent improvements in diagnostic and therapeutic techniques, there has been no improvement in the 5-year survival rates for laryngeal cancer patients over the last 30 years (4). LSCC is a solid tumour and, as with other solid malignancies, progression of the tumour is significantly dependent on angiogenesis (5).

Angiogenesis is a complicated multistep process involving the breakdown of the endothelial cell basement membrane, digestion of the extracellular matrix, proliferation and migration of endothelial cells following the stimulation of angiogenic factors and the formation of new capillaries from pre-existing ones.

The hypothesis that tumours independently produce angiogenic factors was first advanced in the 1960s (6). An association between a greater extent of angiogenesis, a higher incidence of metastases and consequently declining survival rates has been observed in the majority of solid tumours (7). One of the first available studies addressing the role of angiogenesis in laryngeal carcinoma was conducted by Beatrice et al (8) and demonstrated that microvessel count was significant in predicting disease-free survival.

The authors of the present study have already investigated the expression of multiple angiogenic factors in laryngeal carcinoma tissue lysates and corresponding lymph nodes, using an antibody array method that identified an increased expression of several angiogenesis-related factors with progression of the tumour in the laryngeal tissues compared with control uvular tissue (9).

The aim of the present study was to investigate in detail the role of these specific angiogenic factors [angiogenin, insulin-like growth factor binding protein 3 (IGFBP3), vascular endothelial growth factor (VEGF), FGF binding protein (FGF-BP), tissue inhibitor of metalloproteinases-1 (TIMP-1) and interleukin (IL)-8 (IL-8)], which are known to stimulate angiogenesis in head and neck SCC (HNSCC) (10), in a larger sample cohort of laryngeal tumour tissue lysates using ELISA.

\section{Materials and methods}

Patients and samples. Fresh tissue specimens (tumour and/or nodal; Table I) were obtained intra-operatively from 40 patients (9 tumours and corresponding secondary lymph nodes, 27 tumours alone and 4 nodal samples alone) prior to 
chemotherapy and radiotherapy. The patient group comprised 32 males and 8 females, with a mean age of 68 years (range, 51-89 years; Table I).

The study was approved by the Ethics Committees of South Humber Research and Hull and East Yorkshire Hospitals R\&D (06/Q1105/63 and 07/H1305/7) and the patients provided written informed consent.

Preparation of tissue lysates. The tissues were transported to the laboratory in Dulbecco's modified Eagle's medium (DMEM; PAA Laboratories, Somerset, UK), supplemented with $10 \%(\mathrm{v} / \mathrm{v})$ fetal bovine serum (FBS; Biosera, East Sussex, UK), and penicillin/streptomycin (final concentrations: $0.1 \mathrm{U} / \mathrm{ml}$ and $0.1 \mathrm{mg} / \mathrm{ml}$, respectively; PAA). Tissue lysates were prepared by finely mincing the tissue on ice using scalpels in ProteoJET ${ }^{\mathrm{TM}}$ Mammalian Cell Lysis Reagent (500 $\mu \mathrm{l} / 100 \mathrm{mg}$ tissue; Fermentas Life Sciences, York, UK), containing a protease inhibitor cocktail $(1 \mathrm{ml} / 20 \mathrm{~g}$ tissue; Calbiochem/Merck, Nottingham, UK). The lysate was then subjected to sonication for $15 \mathrm{~min}$ on ice prior to centrifugation at $400 \mathrm{x}$ g for $15 \mathrm{~min}$ to pellet cell debris. The resulting supernatant was aliquoted into $0.5-\mathrm{ml}$ lysates and stored at $-80^{\circ} \mathrm{C}$ for protein determination. The protein concentration in each tissue lysate was quantified immediately prior to use in the ELISA using the Coomassie Plus Assay kit (Thermo Scientific, Rockford, IL, USA) following the manufacturer's instructions, in order that the concentration of each factor be standardised per mg of protein.

Determination offactor concentrations with ELISA. Validation of the ELISA for use with tissue lysate was performed by generating standard curves for each factor using both standards diluted in buffer and standards diluted in ProteoJET lysis buffer. No effect of the diluents was observed for any of the factors apart from VEGF. The lysate was dialysed to remove lysis buffer prior to use in the VEGF ELISA using a dialysis cassette (Slide-A-Lyzer G2 Dialysis Cassette 3.5K MCWO; Thermo Scientific), according to the manufacturer's instructions.

The concentration of the angiogenesis-related factors (angiogenin, VEGF, TIMP-1, FGF, IGF, IL-8) present in neat and 1:10 diluted tissue lysates was determined in duplicate using standard sandwich ELISAs (R\&D Systems, Minneapolis, MN, USA) following the manufacturer's instructions. In some cases, lysates were not available to perform all ELISAs and the numbers of lysates investigated are shown in Table II. Assay diluent was included as a blank, followed by absorbance measurement at $450 \mathrm{~nm}$ with wavelength correction at $570 \mathrm{~nm}$ and blank subtraction. Standard curves were generated using a four-parameter logistic curve fit for each factor investigated and used to determine the level of the angiogenic factors present in each lysate.

Statistical analysis. Statistical differences were determined using the statistical package for the social sciences (SPSS) software version 18 (SPSS, Inc., Chicago, IL, USA). The relative expressions of angiogenesis-related proteins were compared between the early (stages T1 and T2) and late (stages T3 and T4) stages of the tumour and between tumour and nodal samples using the Mann-Whitney U test.
Table I. Patient demographics.

\begin{tabular}{|c|c|c|}
\hline Tissue samples & Gender/age (years) & Stage \\
\hline $\begin{array}{l}\text { Tumour only } \\
(n=27)\end{array}$ & $\begin{array}{l}\text { F/89 } \\
M / 65 \\
\text { F/63 } \\
M / 60 \\
M / 72 \\
M / 69 \\
M / 60 \\
M / 71 \\
M / 83 \\
F / 65 \\
M / 69 \\
F / 63 \\
F / 71 \\
M / 74 \\
M / 76 \\
M / 71 \\
M / 52 \\
F / 80 \\
M / 76 \\
M / 68 \\
M / 55 \\
M / 65 \\
F / 81 \\
M / 59 \\
M / 68 \\
M / 51 \\
M / 62\end{array}$ & $\begin{array}{l}\text { T1N0 } \\
\text { T1N0 } \\
\text { T2N0 } \\
\text { T2N0 } \\
\text { T2N2b } \\
\text { T3N0 } \\
\text { T3N1 } \\
\text { T3N2b } \\
\text { T3N2b } \\
\text { T3N2b } \\
\text { T4N0 } \\
\text { T4N0 } \\
\text { T4N0 } \\
\text { T4aN0 } \\
\text { T4aN0 } \\
\text { T4aN0 } \\
\text { T4aN0 } \\
\text { T4aN0 } \\
\text { T4aN0 } \\
\text { T4aN1 } \\
\text { T4aN0 } \\
\text { T4aN0 } \\
\text { T4N1 } \\
\text { T4aN1 } \\
\text { T4aN1 } \\
\text { T4N2b } \\
\text { T4N2c }\end{array}$ \\
\hline $\begin{array}{l}\text { Node only } \\
(n=4)\end{array}$ & $\begin{array}{l}M / 69 \\
M / 71 \\
M / 82 \\
M / 68\end{array}$ & $\begin{array}{l}\text { T1N1 } \\
\text { T1N0 } \\
\text { T2N2a } \\
\text { T3N2b }\end{array}$ \\
\hline $\begin{array}{l}\text { Tumour and } \\
\text { node }(n=9)\end{array}$ & $\begin{array}{l}M / 63 \\
M / 72 \\
M / 55 \\
F / 64 \\
M / 71 \\
M / 67 \\
M / 85 \\
M / 83 \\
M / 59\end{array}$ & $\begin{array}{l}\text { T2N2c } \\
\text { T2N2b } \\
\text { T3N0 } \\
\text { T3N2 } \\
\text { T4N0 } \\
\text { T4N0 }^{a} \\
\text { T4N1 }^{a} \\
\text { T4N1 } \\
\text { T4aN2b }\end{array}$ \\
\hline
\end{tabular}

All patients had moderately differentiated squamous cell carci-

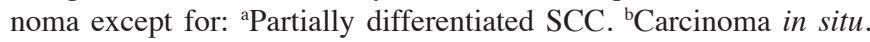
$\mathrm{F}$, female; $\mathrm{M}$, male

\section{Results}

Comparison of angiogenesis-related factor expression between early and late stages of the tumour. Of the 36 tumour samples investigated, 6 samples were from early-stage and 30 from late-stage tumours. Detectable levels of angiogenin were identified in all 6 early-stage tumours investigated and in 26 of the 30 late-stage tumours. Angiogenin demonstrated a significantly higher level of expression in early-stage 
Table II. Comparison of angiogenesis-related factors in lysates from early- and late-stage tumours.

\begin{tabular}{|c|c|c|c|}
\hline Factors & $\mathrm{N}+/ \mathrm{N}$ & Mean \pm SEM & P-value \\
\hline \multicolumn{4}{|c|}{ Angiogenin } \\
\hline Early & $6 / 6$ & $12502.03 \pm 3869.58$ & \multirow[t]{2}{*}{0.033} \\
\hline Late & $26 / 30$ & $4527.05 \pm 952.18$ & \\
\hline \multicolumn{4}{|l|}{ IL-8 } \\
\hline Early & $4 / 5$ & $1255.18 \pm 546.35$ & \multirow[t]{2}{*}{0.330} \\
\hline Late & $22 / 30$ & $852.46 \pm 195.21$ & \\
\hline \multicolumn{4}{|l|}{ IGFBP3 } \\
\hline Early & $2 / 5$ & $8.67 \pm 6.77$ & \multirow[t]{2}{*}{0.314} \\
\hline Late & $23 / 29$ & $35.07 \pm 26.25$ & \\
\hline \multicolumn{4}{|c|}{ FGF-basic } \\
\hline Early & $4 / 5$ & $713.22 \pm 319.05$ & \multirow[t]{2}{*}{0.269} \\
\hline Late & $21 / 29$ & $430.95 \pm 92.95$ & \\
\hline \multicolumn{4}{|l|}{ TIMP-1 } \\
\hline Early & $4 / 5$ & $203.66 \pm 137.46$ & \multirow[t]{2}{*}{0.096} \\
\hline Late & $23 / 28$ & $122.48 \pm 112.47$ & \\
\hline \multicolumn{4}{|l|}{ VEGF } \\
\hline Early & $4 / 4$ & $75.11 \pm 40.08$ & \multirow[t]{2}{*}{0.872} \\
\hline Late & $23 / 30$ & $350.25 \pm 144.41$ & \\
\hline
\end{tabular}

compared to late-stage tumours (Table II). The remaining five angiogenesis-related growth factors (VEGF, FGF-BP, TIMP-1, IL-8 and IGFBP3) were positive in the majority of the tumour lysates investigated. However, they did not exhibit any statistically significant differences between the early and late stages of the tumour (Table II).

Comparison of angiogenesis-related factor expression between tumour and nodal tissue. The final analysis included a total of 36 tumour sample lysates and 13 nodal lysates (nine samples were paired tumour and corresponding nodes). A positive expression was noted in the majority of the lysates of tumour and nodal samples (Table III). IGFBP3 was detectable at significantly higher levels in the tumour compared to nodal lysates (Table III). The remaining five angiogenesis-related factors (VEGF, FGF-BP, TIMP-1, IL-8 and angiogenin)were positive in the majority of the lysates investigated. However, they did not exhibit any statistically significant differences between the tumour and nodal samples (Table III).

\section{Discussion}

A previous pilot study published by the authors (9) investigated the expression of 55 angiogenesis-related factors and demonstrated an increased expression of several proteins associated with tumour progression.

The present study aimed to investigate in detail the expression of six angiogenesis-related growth factors in fresh tissue lysates of laryngeal malignancies and associated metastatic lymph nodes using ELISA. Angiogenin and IGFBP-3 have been identified as factors that may be involved in laryngeal malignancy progression, as angiogenin levels were found to be higher in early-stage compared to late-stage tumours and higher levels of IGFBP3 were detected in tumour tissue lysates compared to the nodal lysates of the laryngeal malignancies. By contrast, VEGF, FGF, IL-8 and TIMP-1, which exhibited detectable levels in tumour lysates, did not exhibit any statistically significant difference between tumour stages or between tumour and nodal tissues.

Previous studies identified VEGF, IL-8 and TIMP-1 as being involved in head and neck cancers. For example. Chen et al (11) investigated the expression of angiogenic factors in tumour tissue, HNSCC cell lines and serum, and established that IL-8, VEGF and GM-CSF produced by HNSCC are consistent with the pathology of the neoplasm. In addition, Ruokolainen et al (12) reported circulating and tissue TIMP-1 to be associated with a shortened cause-specific and relapse-free survival of HNSCC patients. In accordance with the present study, a previous study suggested a role for angiogenin in HNSCC, demonstrating an increased expression measured by immunohistochemistry, in situ hybridization, or serum concentration in numerous malignancies, including breast, cervical, gastric, hepatic, renal, prostate, pancreatic, endometrial and colorectal cancer, leukemia, lymphoma, melanoma and osteosarcoma (13). Moreover, in the majority of these cancers, prognosis has been correlated with the level of angiogenin expression (14).

In contrast to the present investigation, a study by Marioni et al (15) that determined angiogenin expression 
Table III. Expression of angiogenesis-related factors in tumour and nodal tissue.

\begin{tabular}{|c|c|c|c|}
\hline Factors & $\mathrm{N}+/ \mathrm{N}$ & Mean \pm SEM & P-value \\
\hline \multicolumn{4}{|c|}{ Angiogenin } \\
\hline Tumour & $23 / 36$ & $5856 . .21 \pm 1114.2$ & 0.659 \\
\hline Node & $8 / 13$ & $7358.63 \pm 2309.49$ & \\
\hline \multicolumn{4}{|l|}{ IL-8 } \\
\hline Tumour & $26 / 35$ & $910.0 \pm 182.9$ & 0.787 \\
\hline Node & $8 / 13$ & $1682.0 \pm 643.9$ & \\
\hline \multicolumn{4}{|l|}{ IGF-BP3 } \\
\hline Tumour & $25 / 34$ & $31.19 \pm 22.41$ & 0.006 \\
\hline Node & $13 / 13$ & $22.98 \pm 5.01$ & \\
\hline \multicolumn{4}{|l|}{ FGF-basic } \\
\hline Tumour & $25 / 34$ & $472.46 \pm 91.49$ & 0.774 \\
\hline Node & $12 / 13$ & $498.8 \pm 134.1$ & \\
\hline \multicolumn{4}{|l|}{ TIMP-1 } \\
\hline Tumour & $27 / 33$ & $134.78 \pm 97.16$ & 0.329 \\
\hline Node & $13 / 13$ & $17.69 \pm 3.61$ & \\
\hline \multicolumn{4}{|l|}{ VEGF } \\
\hline Tumour & $27 / 34$ & $317.88 \pm 128.16$ & 0.264 \\
\hline Node & $8 / 12$ & $4347.89 \pm 2393.25$ & \\
\hline
\end{tabular}

$\mathrm{N}+$, number of tumours positive for each factor. $\mathrm{N}$, number of lysates available for investigation. Statistical significance shown in bold when Mann-Whitney U test was $\mathrm{P}<0.05$. SEM, standard error of the mean; IL, interleukin; IGFBP, insulin-like growth factor binding protein; FGF, fibroblast growth factor; TIMP, tissue inhibitor of metalloproteinases; VEGF, vascular endothelial growth factor.

immunohistochemically in laryngeal carcinoma cells and intratumoural vessels in a cohort of 108 patients, revealed no correlation with pathological $\mathrm{T}$ and $\mathrm{N}$ stage or grading of the laryngeal cancer. This inconsistency between the two studies may result from the present study including a smaller cohort of patients, with the majority of samples being in the late stage of tumour development, or the different analysis methods employed. Furthermore, we investigated the role of several angiogenic factors, as opposed to the above-mentioned study that investigated role of a single factor. However, Marioni et al (15) reported that angiogenin expression was significantly higher in laryngeal carcinoma samples obtained from patients with locoregional recurrence and disease-free survival was significantly shorter in cases with carcinoma cells exhibiting high angiogenin expression. Therefore, the authors of that study concluded that angiogenin expression in laryngeal cancer may be useful in the detection of laryngeal SCC with a higher risk of disease recurrence. In accordance with the results of the present study, Homer et al (2000) investigated the association of angiogenin, FGF-b and endostatin in the serum of HNSCC patients with prognosis, and observed that angiogenin exhibited a significant negative association with tumour recurrence, poorer grade and advanced stage.

The increased expression of IGFBP3 in the tumour lysates compared to the corresponding nodal lysates of the laryngeal tumours suggested that it may be involved in their progression, as insulin-like growth factors (IGFs) are crucial in regulating cell proliferation and differentiation modulated by a family of high-affinity IGFBPs, of which IGFBP3 is a major serum carrier protein $(16,17)$. Upregulation of IGF signaling via alterations in the expression levels of the ligands, receptors and/or regulatory binding proteins, has been documented in various malignancies. Previous in vitro and in vivo studies suggested the involvement of this signaling axis in tumour initiation and progression $(18,19)$. The majority of the biological actions of IGFs are mediated by the IGF-1R and insulin receptor (IR) tyrosine kinases. A study by Ulanet et al (20), using a transgenic mouse model of pancreatic neuroendocrine carcinogenesis, provided evidence that upregulated IR expression may be functionally associated with tumour progression and demonstrated that the IR is an integral component of the tumour-promoting signaling axis, contributing to tumour progression. The levels of IR were found to be significantly upregulated during the multistep progression from hyperplastic lesion to islet tumour.

Supporting a role for IGFBP3 in HNSCC, Wu et al (21) determined the pre-diagnostic serum IGFBP3 and IGF-1 levels in 80 HNSCC patients who subsequently developed second primary tumours (SPTs) and observed that higher levels of IGF-1 and IGFBP3 were detected in patients who developed SPTs.

A study by Papadimitrakopoulou et al (22) analysed IGFBP3 protein expression by immunohistochemical staining of tissue samples in 34 SCCs of the tongue (stage II-IV) and 30 premalignant lesions of the oral cavity and larynx. The results of that study demonstrated a downregulation of IGFBP3 expression in a significant fraction of patients with stage II-IV tongue SCC and premalignant lesions, as compared to adja- 
cent normal epithelium within the tissue sample that served as a positive control. A different section of the biopsy was used as negative control and was treated with non-immune rabbit the serum, without the addition of the primary antibody. In addition, the authors of that study noted a significant inverse association between the loss of IGFBP3 expression and overall, disease-specific and disease-free survival and they concluded that a low IGFBP3 expression is a predictor of poor prognosis in patients with SCC of the tongue.

VEGF plays a key role in tumour angiogenesis and it has been identified in several malignancies, including HNSCC $(23,24)$. The results of previous studies on VEGF expression and tumour angiogenesis and the association between the clinicopathological factors and prognosis in head and neck tumours, particularly in SCC of the larynx, are controversial $(25,26)$. A previous study by Sullu et al $(27)$ reported a significant association between VEGF expression levels and tumour characteristics; by contrast, another study reported no correlation between VEGF expression and tumour characteristics (25). The different findings may be due to the different methods of detection of VEGF expression and the fact that different studies included tumours from different subsites in the head and neck region, which are known to behave quite differently (27). Despite the conflicting results, we did not observe any significance for VEGF expression.

Our previous pilot study (9) demonstrated a more frequent expression of FGF-BP, TIMP-1 and IL-8 in the tissue lysates compared to the control uvular tissue, although no statistically significant difference was identified in the present study with quantitative analysis between the early and late stages or between the tumour and corresponding nodal tissues. A study by Li et al (28) that investigated the expression of FGF-BP in HNSCC in 35 primary ( 8 from the larynx, 11 from the oral cavity, 4 from the pharynx, 5 from the neck, 3 from the nasal cavity and 4 from the face) and 8 metastatic specimens, as well as 7 control tissues, identified an increased expression of FGF-BP in primary and metastatic tissue specimens compared to adjacent control tissue and concluded that an increased FGF-BP expression is associated with angiogenesis by measuring microvessel count. Ruokolainen et al (12) observed that pre-treatment serum levels of TIMP-1 (n=68) and TIMP-1 in the tumour tissue $(n=74)$ were positively correlated with the size of the tumour. However, in accordance with the present findings, no positive correlation was observed between tissue TIMP-1 positivity or serum TIMP-1 levels and disease stage or tumour grade. Druzgal et al (29) reported that the serum levels of IL-6, IL-8, hepatocyte growth factor and VEGF were increased in patients with HNSCC as compared to the normal control group. However, no association was observed between IL-8 levels and tumour stage in the present study or between tumour and metastatic nodes. The inconsistency between our results and those of other studies may be due to the fact that our study included fresh samples from a single subsite (larynx), whereas the majority of the studies in the literature included multiple HNSCC subsites and investigated serum rather than intratumoural levels.

In conclusion, to the best of our knowledge, this study is the first to investigate the role of multiple angiogenic factors in tissue lysates of tumours and corresponding metastatic lymph nodes and identified angiogenin and IGFBP3 as being poten- tially involved in the development of laryngeal malignancies. Further studies are required to elucidate the function of these factors before a significant role for these factors is ascertained.

\section{Acknowledgements}

The authors would like to thank Professor John Greenman and V. L. Green for the their continuous support in completing this study.

\section{References}

1. Cooper JS, Porter K, Mallin K, et al: National Cancer Database report on cancer of the head and neck: 10-year update. Head Neck 31: 748-758, 2009.

2. Almadori G, Bussu F, Cadoni G, Galli J, Paludetti G and Maurizi M: Molecular markers in laryngeal squamous cell carcinoma: towards an integrated clinicobiological approach. Eur J Cancer 41: 683-693, 2005.

3. Krecicki T, Zalesska-Krecicka M, Jagas M, Szajowski K and Rak J: Laryngeal cancer in Lower Silesia: descriptive analysis of 501 cases. Oral Oncol 34: 377-380, 1998.

4. Jemal A, Siegel R, Ward E, Murray T, Xu J, Smigal C and Thun MJ: Cancer statistics, 2006. CA Cancer J Clin 56: 106-130, 2006.

5. Folkman J: Angiogenesis in cancer, vascular, rheumatoid and other disease. Nat Med 1: 27-31, 1995.

6. Carmeliet P and Jain RK: Angiogenesis in cancer and other diseases. Nature 407: 249-257, 2000.

7. Des Guetz G, Uzzan P, Nicolas P, et al: Microvessel density and VEGF expression are prognostic factors in colorectal cancer. Meta-analysis of the literature. Br J Cancer 94: 1823-1832, 2006.

8. Beatrice F, Valente R, Cammarota R, et al: Laryngeal cancer and angiogenesis. Acta Otorhinolaryngol Ital 16: 355-362, 1996

9. Korampalli TS, Green VL, Greenman J and Stafford ND: Protein profiling of angiogenesis-related growth factors in laryngeal carcinoma: Pattern of protein expression in relation to tumour progression. Int J Oncol 39: 1033-1039, 2011.

10. Bergers $G$ and Benjamin LE: Tumorigenesis and the angiogenic switch. Nat Rev Cancer 3: 401-410, 2003.

11. Chen Z, Malhotra PS, Thomas GR, et al: Expression of proinflammatory and proangiogenic cytokines in patients with head and neck cancer. Clin Cancer Res 5: 1369-1379, 1999.

12. Ruokolainen H, Paakko P and Turpeenniemi-Hujanen T: Tissue inhibitor of matrix metalloproteinase-1 is prognostic in head and neck squamous cell carcinoma: comparison of the circulating and tissue immunoreactive protein. Clin Cancer Res 11: 3257-3264, 2005

13. Gao X and Xu Z: Mechanisms of action of angiogenin. Acta Biochim Biophys Sin (Shanghai) 40: 619-624, 2008.

14. Katona TM, Neubauer BL, Iversen PW, Zhang S, Baldeidge LA and Cheng L: Elevated expression of angiogenin in prostate cancer and its precursors. Clin Cancer Res 11: 8358-8363, 2005.

15. Marioni G, Marioni F, Blandamura S, et al: Neoangiogenesis in laryngeal carcinoma: angiogenin and CD105 expression is related to carcinoma recurrence rate and disease-free survival. Histopathology 57: 535-543, 2010.

16. Baxter RC: Signalling pathways involved in antiproliferative effects of IGFBP-3: a review. Mol Pathol 54: 145-148, 2001.

17. Stewart CE and Rotwein P: Growth, differentiation, and survival: multiple physiological functions for insulin-like growth factors. Physiol Rev 76: 1005-1026, 1996.

18. Samani AA, Yakar S, LeRoith D and Brodt P: The role of the IGF system in cancer growth and metastasis: overview and recent insights. Endocr Rev 28: 20-47, 2007.

19. Pollak MN, Schernhammer ES and Hankinson SE: Insulin-like growth factors and neoplasia. Nat Rev Cancer 4: 505-518, 2004.

20. Ulanet DB, Ludwig, DL, Kahn CR and Hanahan D: Insulin receptor functionally enhances multistage tumour progression and conveys intrinsic resistance to IGF-1R targeted therapy. Proc Natl Acad Sci USA 107: 10791-10798, 2010.

21. Wu X, Zhao H, Do KA, Johnson MM, Dong Q, Hong WK and Splitz MR: Serum levels of insulin growth factor (IGF-I) and IGF-binding protein predict risk of second primary tumours in patients with head and neck cancer. Clin Cancer Res 10: 3988-3995, 2004. 
22. Papadimitrakopoulou VA, Brown EN, Liu DD, El-Naggar AK Jack Lee J, Hong WK and Lee HY: The prognostic role of loss of insulin-like growth factor-binding protein-3 expression in head and neck carcinogenesis. Cancer Lett 239: 136-143, 2006.

23. Shang ZJ, Li ZB and Li JR: VEGF is up-regulated by hypoxic stimulation and related to tumour angiogenesis and severity of disease in oral squamous cell carcinoma: in vitro and in vivo studies. Int J Oral Maxillofac Surg 35: 533-538, 2006.

24. Ferrara N: Vascular endothelial growth factor. Eur J Cancer 32A: 2413-2422, 1996.

25. Tae K, El-Naggar AK, Yoo E, et al: Expression of vascular endothelial growth factor and microvessel density in head and neck tumourigenesis. Clin Cancer Res 6: 2821-2828, 2000.

26. Bandoh N, Hayashi T, Takahara M, et al: VEGF and bFGF expression and microvessel density of maxillary sinus squamous cell carcinoma in relation to p53 status, spontaneous apoptosis and prognosis. Cancer Lett 208: 215-225, 2004.
27. Sullu Y, Gun S, Atmaca S, Karagoz F and Kandemir B: Poor prognostic clinicopathologic features correlate with VEGF expression but not with PTEN expression in squamous cell carcinoma of the larynx. Diagn Pathol 5: 35, 2010.

28. Li W, Wang C, Juhn SK, Ondrey FG and Lin J: Expression of fibroblast growth factor binding protein in head and neck cancer. Arch Otolaryngol Head Neck Surg 135: 896-901, 2009.

29. Druzgal CH, Chen Z, Yeh NT, et al: A pilot study of longitudinal serum cytokine and angiogenesis factor levels as markers of therapeutic response and survival in patients with head and neck squamous cell carcinoma. Head Neck 27: 771-784, 2005. 